\title{
A Polynomial Time Solution to Minimum Forwarding Set Problem in Wireless Networks under Unit Disk Coverage Model
}

\author{
Mehmet Baysan, Student Member, IEEE, Kamil Sarac, Member, IEEE, R. Chandrasekaran and Sergey Bereg
}

\begin{abstract}
Network-wide broadcast (simply broadcast) is a frequently used operation in wireless ad hoc networks. One promising practical approach for energy efficient broadcast is to use localized algorithms to minimize the number of nodes involved in the propagation of the broadcast messages. In this context, the minimum forwarding set problem (MFSP) (also known as multi-point relay (MPR) problem) has received a considerable attention in the research community. Even though the general form of the problem is shown to be NP-complete, the complexity of the problem has not been known under the practical application context of ad hoc networks. In this paper, we present a polynomial time algorithm to solve the MFSP problem for wireless network under unit disk coverage model. We prove the existence of some geometrical properties for the problem and then propose a polynomial time algorithm to build an optimal solution based on these properties. To the best of our knowledge, our algorithm is the first polynomial time solution to the MFSP problem under the unit disk coverage model. We believe that the work presented in this paper will have an impact on the design and development of new algorithms for several wireless network applications including energy efficient multicast, broadcast, and topology control protocols for wireless ad hoc networks and sensor networks.
\end{abstract}

Keywords: Multi-point relays, minimum forwarding set problem, network wide broadcast, unit disk graphs.

\section{INTRODUCTION}

Wireless ad hoc networks (WANETs) are used to provide communication services in dynamic environments including active battlefield, search and rescue, and emergency relief. Energy and wireless bandwidth are two scarce WANET resources that need to be used efficiently. Energy is limited as the nodes typically operate on battery power. Wireless bandwidth is limited as the nodes share the same transmission medium which is open to collision and contention. Network-wide broadcast (simply broadcast) is a frequently used operation in WANETs. In addition to data dissemination, many protocols utilize broadcast to communicate control messages [1], [2], [3], [4]. As an example, popular WANET routing protocols, including OLSR, AODV, and DSR, use broadcast to discover and maintain routes between the nodes in a WANET. A naive implementation of the broadcast operation where each node involves in propagation of a broadcast message (i.e., network wide flooding) may cause a high level of energy and bandwidth consumption in WANETs [5].

Mehmet Baysan is with Dept. of Management at Univ. Toronto. All the other authors are with Dept. of Computer Science at Univ. Texas at Dallas (emails: baysan@gmail.edu,ksarac@utdallas.edu,chandra@utdallas.edu,and besp@utdallas.edu) (At the time this paper was written, Mehmet Baysan was with Univ. Texas at Dallas)

\section{A. Problem Definition}

Energy efficient broadcast problem received a significant attention from the research community and a large number of studies have been published in the area [6]. One promising approach that was proposed for energy efficient broadcast is the neighbor designation approach [7] where the goal is to prevent unnecessary transmission of broadcast packets for energy efficiency. Each node collects 2-hop neighborhood information and then identifies a subset of its 1-hop neighbors as forwarding nodes for relaying a broadcast message toward its 2-hop neighbors. The efficiency of neighbor designation approach depends on finding a minimum size forwarding node set among the 1-hop neighbors. This problem is referred to as Minimum Forwarding Set Problem (MFSP) [7], [8] and is formally defined as follows:

Definition 1 (Minimum Forwarding Set Problem (MFSP)): Consider a graph $G=(V, E)$ where $V$ is the set of nodes and $E$ is the set of links in the network. Given a node $v \in V$, let $N(v)$ and $N_{2}(v)$ represent the set of 1-hop and 2-hop neighbors of $v$, respectively. $N(v)$ and $N_{2}(v)$ are strict sets such that $v \notin N(v)$ and $N(v) \cap N_{2}(v)=\varnothing$. MFSP asks for a minimum-size subset $S$ of $N(v)$ such that every node in $N_{2}(v)$ is within the coverage of at least one node in $S$. More formally, MFSP asks for a minimum cardinality set $S$ such that $S \subseteq N(v)$ and $\left(\forall x \in N_{2}(v), \exists y \in S \mid x \in N(y)\right)$.

A solution to the MFSP problem at a node $v$ is $S \subseteq N(v)$ where $S$ is a minimum cardinality set called forwarding set. Note that in an optimal solution, the assignment of a node $b \in N_{2}(v)$ to a node $s \in S$ requires that $b \in N(s)$. In other words, in the context of the wireless broadcast operation, $b$ should be within the coverage range of $s$. Also note that, in certain cases, multiple different optimal solutions may exist.

\section{B. Existing Solutions}

The MFSP problem is shown to be NP-complete [7] with a reduction from the Set Cover problem. The heuristic proposed in [7] is an application of the well-known Chvatal's greedy algorithm for the Set Cover problem [9] and gives an approximation ratio of $\left(1+\ln \left(\left|S_{i}\right|_{\max }\right)\right)$ where $\left|S_{i}\right|_{\max }$ is the size of the largest subset of $N_{2}(v)$ that is covered by a node $i \in N(v)$. Busson et al. [10] presented a stochastic analysis to argue that the heuristic in [7] performs near optimal for most practical scenarios.

Calinescu et al. [8] studied the problem under the assumption that nodes are distributed in 2-dimensional plane and they 
have a a unit disk coverage [11] for their transmissions. They proposed a 6-approximation algorithm that runs in $O(n \log n)$ time and a 3-approximation algorithm that runs in $O\left(n \log ^{2} n\right)$ time. In addition, they presented an exact $O\left(n \log ^{2} n\right)$ time algorithm for a special case of the MFSP problem when all 2-hop neighbors are in the same quadrant of a 2-dimensional coordinate space with respect to the broadcasting node.

Finally, $\mathrm{Wu}$ et al. [12] considered an extended version of the MFSP problem where a broadcasting node $v$ collects 3-hop neighbor information to find a small number of 1-hop and/or 2-hop neighbors to cover the set of 2-hop neighbors. They proposed a heuristic that gives a constant local approximation ratio to identify an extended forwarding node set. We believe that the extended version of the problem introduces a new dimension to the original problem setup with some potential performance improvements depending on the availability of 3-hop neighborhood information. In this paper, we consider the original MFSP problem as defined above and leave the extended version of the problem for future work.

\section{Our Contributions}

In this paper, we present the first polynomial time algorithm to solve the MFSP problem under unit disk coverage model for wireless transmission. First, we introduce two properties named as Two-Set Property and Non-Interleaving Property. We then present an algorithm that uses a dynamic programming approach to build an optimal solution and prove its correctness. The algorithm has $O\left(n^{3}+n^{2} m\right)$ time complexity where $m=|N(v)|$ and $n=\left|N_{2}(v)\right|$ for a broadcasting node $v$. The current version of our algorithm works under the unit disk coverage model and therefore may have limited utility for real world wireless networks. However, the algorithm can be quite instrumental in evaluating the performance of more practical heuristics within simulation studies.

The rest of the paper is organized as follows. The next section is on the related work. Section III presents the problem setup and establishes some facts about intersecting unit disks. Section IV introduces the two geometric properties that we utilize in our algorithm. Section V presents our polynomial time exact algorithm and Section VI concludes the paper.

\section{RELATED WORK}

The MFSP problem emerged within the context of network wide broadcast in WANETs. In this section, we present a brief summary of the related problems and refer our readers to [13], [14], [15] for more information on the existing literature on energy efficient broadcast operation in WANETs. The general case of the MFSP problem is an instance of the well-known NP-complete Set Cover problem [7]. Set Cover problem has been extensively studied in the literature and early approximation algorithms have been proposed for both unweighted version by Johnson [16] and by Lovasz [17], and for weighted version by Chvatal [9]. These algorithms give an approximation ratio of $1+\ln (\Delta)$ where $\Delta$ is the cardinality of the maximum cardinality subset $\left(\max _{i \leq n}\left|S_{i}\right|\right)$. In [18], Hochbaum presents an algorithm for the weighted version with an approximation ratio of $\alpha$ where $\alpha$ represents the maximum number of subsets covering an element. The running time of this algorithm is $O\left(n^{3}\right)$. In [19], Bar-Yehuda and Even present an algorithm with a similar approximation ratio but an improved running time of $O\left(n^{2}\right)$. We refer readers to [20] for other approximation algorithms on the Set Cover problem.

The MFSP problem becomes a geometrical problem when we use unit disks to model the coverage area of wireless transmissions. Unit disk graphs (UDGs) are neither perfect nor planar graphs [11]. Thus, efficient algorithms proposed for planar and perfect graphs cannot be applied to UDGs. MFSP problem under the unit disk coverage assumption resembles to the well-known Minimum Dominating Set (MDS) problem. MDS problem for UDGs has been studied extensively. The problem is shown to be NP-complete for UDGs [11]. In [21], Marathe et al. present a linear time approximation algorithm with a constant-factor performance guarantee of 5. In [22], a polynomial-time approximation scheme (PTAS) with $((k+$ $1) / k)^{2}$ guarantee is given for a constant $k$ in $n^{O\left(k^{3}\right)}$. Minimum Connected Dominating Set (MCDS) problem is a different version of the problem in which the dominating set should be connected. In [23], Cheng et al. presented a PTAS for MCDS problem. In [24], Ambuhl et al. presented constantfactor approximation algorithms for the weighted versions of MDS and MCDS problems. These approximations do not apply to MFSP problem as the dominating nodes in MFSP should be chosen from only 1-hop neighbors.

Another related problem to MFSP problem is covering with disks which aims at finding a minimally sized set of unit disks to cover given points on the plane (disks can be placed arbitrarily). This problem is examined in [25] and a $O\left(l^{2}(l * \sqrt{2})^{2} \cdot(2 n)^{2(l \sqrt{2})^{2}+1}\right)$ time approximation algorithm is given with a performance guarantee of $(1+1 / l)^{2}$. The difference between this problem and our problem is in the selection of the disks. This problem selects arbitrary disks to cover given points, but in our problem we are bound to select disks from the set of on-hop neighboring nodes.

Another related problem to MFSP problem is the well known Disk Cover (DC) problem that tries to find a minimal size set of disks (from a given set of disks) to cover a given set of points on a plane [25]. In [26], authors present an algorithm with an approximation ratio of $O(1)$ and running time of $O\left(c^{2} n \log n \log (n / c)\right)$ where $c$ represents the size of the optimal solution. MSFP problem is a special instance of the DC problem where disks are selected from a given set of 1-hop nodes.

Another related work in the context of wireless broadcast is Localized Broadcast Incremental Power Protocol (LBIP) [27]. In LBIP, nodes are assumed to have variable transmission power and the goal is to cover 2-hop neighbors with minimum energy. LBIP involves selection of forwarding nodes as well as determining transmission power levels for such nodes to achieve minimum energy usage. In our current work, we assume fixed transmission power (i.e., unit disk coverage) and our goal is to choose a minimum number of 1-hop neighbors to cover all 2-hop neighbors.

Finally, the most related work to our study in this paper is the previous work by Calinescu et al. [8]. In their work, they propose approximation algorithms to solve the MFSP 
problem (see Section I-B). In this paper, we use a similar setup and develop the first polynomial time algorithm for minimum forwarding node selection.

\section{PRELIMINARIES}

\section{A. The Practical Setup of the Problem}

Most studies use a unit disk or a sphere to represent the shape of the effective coverage area of wireless transmissions [28]. This assumption, though may not always hold in practice, helps in gaining more insight to the problem within the practical context of wireless transmissions. In this paper we consider a similar setup and assume a unit disk coverage model for wireless transmissions. In addition, as most local knowledge based broadcast approaches [6], our approach requires the availability of 2-hop neighborhood information. The required information includes (1) the identities of the 1hop and 2-hop neighbors and (2) a radial ordering (which we define in Section III-B) of the 2-hop neighbors with respect to the broadcasting node. The availability of the position information for the nodes is sufficient to compute the radial ordering of the 2-hop neighbors. One simple way of acquiring the position information is to use a GPS unit at each node. Another possibility is to use the distance and angle information between the neighboring nodes. The distance information can be calculated by using the transmission and reception power level within an energy consumption model [29] that is representative for the environment. The angle information between neighboring nodes can be measured by using multiple ultrasound receivers or directional antennas. Recently, Calinescu [30] proposed methods to calculate 2-hop neighborhood information (identities and positions) for the cases where GPS or distance and angle information is available with a message complexity of $O(n)$ where $n$ is the total number of the nodes in the network.

\section{B. Definitions}

Consider an instance of MFSP problem at a node $v$. Let $N(v)$ and $N_{2}(v)$ represent the 1-hop and 2-hop neighbors of $v$, respectively. To avoid the introduction of excessive notation, let $v$ also represent the location of the node $v$ in a 2-dimensional space. Similarly, let each set $N(v)$ and $N_{2}(v)$ represent the set of points that 1-hop and 2-hop neighbors of $v$ are located in 2-dimensional space. The coverage area of node $v$ is a unit disk (i.e., a disk with a origin at point $v$ and radius $r_{1}=1$ ) represented by $D_{v}$. Let $\bar{D}_{v}$ represent the area of the annulus with an origin at point $v$ and radii $r_{1}=1$ and $r_{2}=2$, i.e., $\bar{D}_{v}=A\left(v, r_{2}\right) \backslash D_{v}$ where $A\left(v, r_{2}\right)$ is a disk with origin at point $v$ and radius $r_{2}=2$ (see Figure 1-a). By this definition, we have $N(v) \subseteq D_{v}$ and $N_{2}(v) \subseteq \bar{D}_{v}$. Based on this setup, we introduce several definitions below.

Definition 2 (Radial Order): Radial order is the ordering of a set of points in $\bar{D}_{v}$ (or the nodes at those points) by using the angle that they make with the origin (point) $v$. Radial order is a cyclic order. If two or more points make the same angle with $v$, then their distance to $v$ can be used to put them into a total order.
Consider the example scenario in Figure 1-(a) where $N_{2}(v)=$ $\{a, b, c, d, e\}$. Starting from the exact south position, the nodes in $N_{2}(v)$ form a radial order as $(e<d<c<b<a)$. The geometrical properties introduced below and the algorithm presented in Section V-A use the radial ordering of the nodes in $N_{2}(v)$ in finding an optimal solution. As we discussed in Section III-A, a node $v$ can compute the radial ordering of the nodes in $N_{2}(v)$ from the collected geographical location information from its neighbors. Therefore, from now on we assume that the radial ordering of the nodes in $N_{2}(v)$ is known by $v$.

Definition 3 (Radially Continuous Neighbor (RCN) Interval): One or more points in the area $\bar{D}_{v}$ that form a continuous interval in the radial order with respect to (w.r.t.) $v$ are said to form a radially continuous neighbor $(\mathrm{RCN})$ interval.

As an example, in Figure 1-(a), $(a>b>c)$ and $(e>a>b)$ form RCN intervals w.r.t. $v$ but $(a>b>d)$ does not as $c \in N_{2}(v)$ separates this interval into two non-consecutive intervals.

Definition 4 (Radially Continuous Coverage Area (RCCA)): Consider a set $S \subseteq N(v)$. For a node $s \in S$, RCCA of $s$ is a continuous subarea in $\bar{D}_{v}, \operatorname{RCCA}(\mathrm{s}) \subseteq \bar{D}_{v}$, such that $s$ is the only node in $S$ that can cover all the points in RCCA(s). A node $s \in S$ may have zero or more RCCAs.

Definition 5 (Connectivity Matrix): Consider an instance of MFPS problem at a node $v$. Let $N(v)=\left\{b_{1}, b_{2}, \ldots, b_{m}\right\}$ and $N_{2}(v)=\left\{a_{1}, a_{2}, \ldots, a_{n}\right\}$ be the 1-hop and 2-hop neighbors of $v$ respectively. A connectivity matrix $R$ is an $m \times n$ matrix that shows the connectivity relation between the nodes in $N(v)$ and $N_{2}(v)$. For a given $b_{i} \in N(v)$ and $a_{j} \in N_{2}(v), R_{i, j}=1$ if $a_{j} \in N\left(b_{i}\right)$ and $R_{i, j}=0$ otherwise.

Definition 6 (Coverage Matrix): Let $m=|N(v)|$ and $n=$ $\left|N_{2}(v)\right|$ for a node $v$. Using two hop neighborhood information, $v$ generates a coverage matrix as a $m \times n$ matrix $C$. Each row in $C$ corresponds to a 1-hop neighbor of $v$ and each column corresponds to a 2-hop neighbor of $v$. An entry $C_{e f}=\left(a_{p}, \bar{p}\right)$ represents the longest RCN interval in $N_{2}(v)$ that is covered by $e \in N(v)$ and that includes $f$ in it. If $f \notin N(e)$, then $C_{\text {ef }}=\varnothing$.

Please see Figure 2 for an example of connectivity and coverage matrices for a node $v$.

Definition 7 (Maximum Coverage Interval (MCI)): An MCI of a node $s \in N(v)$ is an RCN interval $\left\{a_{i}, \ldots, a_{j}\right\} \in N_{2}(v)$ that is completely covered by $s$ such that $s$ cannot cover neither of $a_{i-1}$ and $a_{j+1}$. Note that $s$ can have multiple MCIs in $N_{2}(v)$.

Definition 8 (Essential Coverage): Consider a node $s \in$ $S \subseteq N(v)$ that covers a point $a_{i} \in \bar{D}_{v} . s$ is said to be essential to cover $a_{i}$ if no other node $t \in S$ covers $a_{i}$. If $s$ is essential for a node (at a point) $a_{i}$ in an MCI that it covers, than $s$ is essential for this MCI. Similarly, a node $s \in S \subseteq N(v)$ is said to be essential to cover an $\mathrm{RCCA}(\mathrm{s})$.

Note that the essentiality of $s$ in $\bar{D}_{v}$ is w.r.t. $S \subseteq N(v)$.

\section{Intersection Characteristics of Two Unit Disks}

Consider a unit disk centered at a point $v$. Let $D_{v}$ represent the unit disk and the area covered by it and $C_{v}$ represent 


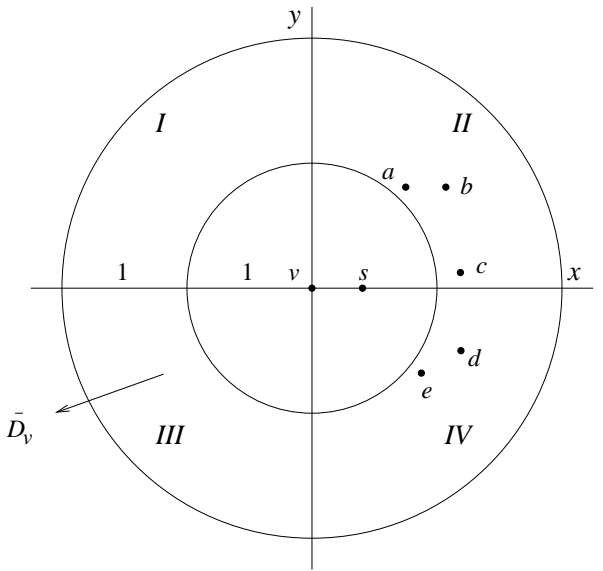

(a)

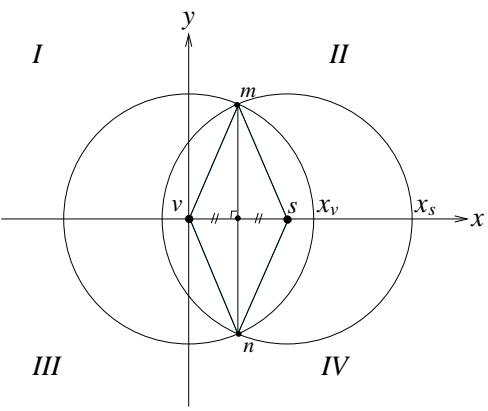

(b)

Fig. 1. Some geometric relations of two intersecting unit disks.

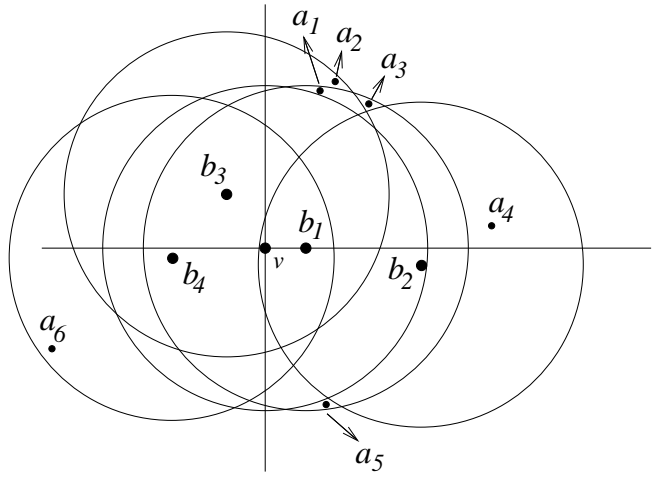

Fig. 2. An MFPS instance at $v$ and its connectivity and coverage matrices.

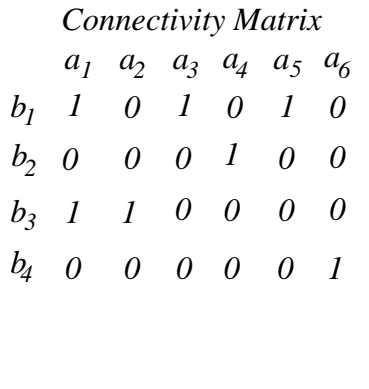

Connectivity Matrix

$\begin{array}{lllllll}b_{1} & 1 & 0 & 1 & 0 & 1 & 0\end{array}$

$\begin{array}{lllllll}b_{2} & 0 & 0 & 0 & 1 & 0 & 0\end{array}$

$\begin{array}{lllllll}b_{3} & 1 & 1 & 0 & 0 & 0 & 0\end{array}$

$\begin{array}{lllllll}b_{4} & 0 & 0 & 0 & 0 & 0 & 1\end{array}$

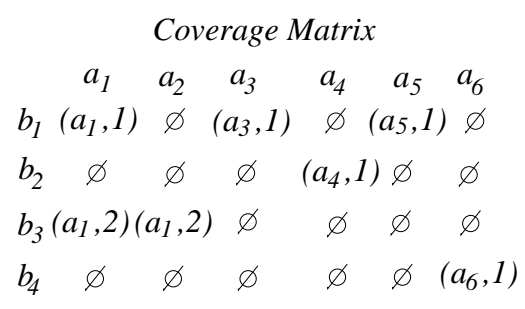

Coverage Matrix

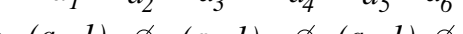

$b_{1}\left(a_{1}, 1\right) \varnothing \quad\left(a_{3}, 1\right) \quad \varnothing\left(a_{5}, 1\right) \varnothing$

$b_{2} \quad \varnothing \quad \varnothing \quad \varnothing \quad\left(a_{4}, 1\right) \varnothing \quad \varnothing$

$b_{4} \quad \varnothing \quad \varnothing \quad \varnothing \quad \varnothing \quad \varnothing\left(a_{6}, 1\right)$ the circle enclosing $D_{v}$. Assume that $v$ is at the origin of a two-dimensional space which is divided into four sub-spaces, named as $I, I I, I I I$, and $I V$, by the $x$ and $y$ coordinate axes as shown in Figure 1-(a). Consider a second point $s$ that is on the $x$-axis to the east of $v$. Similar to the case for $v$, let $D_{s}$ and $C_{s}$ represent the unit disk and the enclosing circle for $s$.

When $|v s|=2, C_{v}$ and $C_{s}$ are tangent to each other and $D_{v}$ and $D_{s}$ intersect at a single point. When $|v s|<2, C_{v}$ and $C_{s}$ intersect twice and $D_{v} \cap D_{s} \neq \varnothing$ as shown in Figure 1(b). Let $m$ and $n$ represent the intersection points of $C_{v}$ and $C_{s}$. Note that, since $s$ is on $x$-axis to the east of $v, m$ and $n$ have to be to the east of $y$-axis. This intersection forms two equal angles as $\widehat{m v n}$ and $\widehat{m s n}$. Let $\alpha$ represent these two equal angles. When $|v s| \leq 1, \alpha$ is in $\left[\frac{2 \pi}{3}, \pi\right]$ and when $1<|v s| \leq 2$, $\alpha$ is in $\left[0, \frac{2 \pi}{3}\right)$. Also note that when $|v s| \leq 1$, the length of the arc $\operatorname{arc}\left(m x_{v} n\right)$ (the segment of $C_{v}$ corresponding to $\alpha$; here $x_{v}$ is the intersection of $C_{v}$ with $x$ axis) is in $\left[\frac{2 \pi}{3}, \pi\right]$. Similarly, the length of the $\operatorname{arc} \operatorname{arc}\left(m x_{s} n\right)$ (the segment of $C_{s}$ corresponding to $2 \pi-\alpha$; here $x_{s}$ is the intersection of $C_{s}$ with $x$ axis) is in $\left[\pi, \frac{4 \pi}{3}\right]$. Finally, the line segment connecting $m$ and $n$ vertically is referred to as Chord $_{v s}$ and it divides the line segment between $v$ and $s$ into two equal parts.

\section{Intersection Characteristics of Three Unit Disks}

In this section, we consider the intersection characteristics of three unit disks in a special setup that is relevant to the MFSP problem. Consider an instance of MFSP problem at a node $v$ and consider $\{s, t\} \in S \subseteq N(v)$. Similar to the above discussion, we assume that $v$ defines a two-dimensional coordinate space and $s$ lies to the exact east of $v$ in this coordinate space. Note that $s$ and $t$ are neighbors of $v$, and $|v s| \leq 1,|v t| \leq 1$, and $|s t| \leq 2$. For $|s t|<2, C_{s}$ and $C_{t}$ intersect twice and $D_{s} \cap D_{t} \neq \varnothing$. and for $|s t|=2, C_{s}$ and $C_{t}$ are tangent to each other, $D_{s}$ and $D_{t}$ intersect at a single point. Observe that since $s$ lies to the east of $v$, the coverage area $D_{s}$ beyond $D_{v}$ (i.e., $D_{s / v}=D_{s} \backslash D_{v}$ ) lies in region $(I I \cup I V)$. Similarly, let $D_{t / v}$ represent the coverage area $D_{t}$ beyond $D_{v}$, i.e., $D_{t / v}=D_{t} \backslash D_{v}$. In addition, let $C_{s / v}$ and $C_{t / v}$ represent the segments of $C_{s}$ and $C_{t}$ outside the coverage area $D_{v}$, respectively.

From MFSP problem's point of view, we study the nature of the coverage area in $D_{s / v}$ that $s$ is essential for (i.e., number and nature of RCCA(s) w.r.t. $S$ ) in the presence of intersections that $C_{s / v}$ may have with $C_{t / v}$. Note that $C_{s / v}$ and $C_{t / v}$ can have zero, one, or two intersections with each other.

Lemma 1: Let $S=\{s, t\} \subseteq N(v)$ in an instance of MFSP problem at $v$. If $C_{s / v}$ has no intersection with $C_{t / v}$, then 
$D_{s / v} \cap D_{t / v}=\varnothing$.

Proof of Lemma 1: Let the intersection points of $C_{v}$ and $C_{s}$ be $m$ and $n$. Let $x_{v}^{+}$and $x_{s}^{+}$be the points that $C_{v}$ and $C_{s}$ intersect $\mathrm{x}$-axis on east of $v$, respectively (see Figure 3-(a)). Given that $C_{s / v}$ and $C_{t / v}$ have zero intersection, $C_{t}$ and $C_{s}$ intersect twice in $D_{v}$. Since $D_{t}$ and $D_{v}$ are both unit disks, $C_{t}$ cannot be enclosed in $D_{v}$. Therefore, $C_{t}$ should intersect $C_{v}$ at two points, say $\bar{m}$ and $\bar{n}$. While tracing $C_{t}$ clockwise direction, assume $\bar{m}$ is the point $C_{t}$ enters into $D_{v}$ and $\bar{n}$ the point that $C_{t}$ exits $D_{v}$. Observe that $\bar{m}$ and $\bar{n}$ cannot be to east of Chord $_{v s}$ (i.e., the line crossing $m$ and $n$ ) as otherwise $C_{t}$ intersects $C_{s / v}$. Assume now that $D_{s / v} \cap D_{t / v} \neq \varnothing$. Since $C_{t}$ cannot intersect $C_{s / v}$, this is only possible if $C_{t}$ intersects $\operatorname{arc}\left(m x_{s}^{+} n\right)$ twice where $m$ and $n$ are the intersection points of $C_{v}$ and $C_{s}$. But, since $C_{v}$ and $C_{t}$ already intersected at $\bar{m}$ and $\bar{n}$ on west of Chord $_{v s}$, they cannot intersect on $\operatorname{arc}\left(m x_{s}^{+} n\right)$. Hence $D_{s / v} \cap D_{t / v} \neq \varnothing$ is not possible.

Corollary 1: Let $S=\{s, t\} \subseteq N(v)$ in an instance of MFSP problem at $v$. If $C_{s / v}$ has no intersection with $C_{t / v}$, then $D_{s / v}$ is an RCCA of s w.r.t. $S$.

Lemma 2: Let $S=\{s, t\} \subseteq N(v)$ in an instance of MFSP problem at $v$. If $C_{s / v}$ has one intersection with $C_{t / v}$, then $s$ is essential to cover one single RCCA in $D_{s / v}$ w.r.t. $S$.

Proof of Lemma 2: From the previous section, the intersection of two disks $D_{s}$ and $D_{t}$ results in three coverage areas as (1) $D_{s} \backslash D_{t}$, (2) $D_{t} \backslash D_{s}$, and (3) $D_{s} \cap D_{t}$. We consider the parts of these coverage areas in $\bar{D}_{v}$ namely $D_{s / v / t}=\left(D_{s} \backslash D_{t}\right) \cap \bar{D}_{v}, D_{t / v / s}=\left(D_{t} \backslash D_{s}\right) \cap \bar{D}_{v}$, and $D_{s t / v}=\left(D_{s} \cap D_{t} \cap \bar{D}_{v}\right)$, respectively (see Figure 3-(b)). Let $p$ be the intersection point of $C_{s / v}$ and $C_{t / v}$. Consider a line $l$ that originates at $v$ and crosses $p$ as in Figure 3-(b). The line $l$ divides $\left(D_{s / v} \cup D_{t / v}\right) \cap \bar{D}_{v}$ into two areas such that the radial order of the points at both sides of $l$ are disjoint from each other. In this case, $s$ is essential to cover one RCCA that includes $D_{s / v / t}$ and part of $D_{s t / v}$ below line $l$ and $t$ is essential to cover one RCCA that includes $D_{t / v / s}$ and part of $D_{s t / v}$ above line $l$ as in Figure 3-(b).

Lemma 3: Let $S=\{s, t\} \subseteq N(v)$ in an instance of MFSP problem at $v$. If $C_{s / v}$ has two intersections with $C_{t / v}$, then $s$ is essential to cover one or two RCCAs in $D_{s / v}$ w.r.t. $S$.

Proof of Lemma 3: If $C_{t / v}$ and $C_{s / v}$ intersect twice, $C_{t}$ cannot intersect $C_{s}$ in $D(v)$. Let $m_{1}$ and $n_{1}$ be the intersection points of $C_{t / v}$ and $C_{s / v}$ and $\bar{m}$ and $\bar{n}$ be the intersection points of $C_{v}$ and $C_{t}$. We have two cases: (1) both $\bar{m}$ and $\bar{n}$ are on $\operatorname{arc}\left(m x_{v}^{+} n\right)$ or (2) both $\bar{m}$ and $\bar{n}$ are on $\operatorname{arc}\left(m x_{v}^{-} n\right)$. Assume the contrary that $\bar{m}$ (or $\bar{n}$ ) is on $\operatorname{arc}\left(m x_{v}^{+} n\right)$ and $\bar{n}$ (or $\left.\bar{m}\right)$ is on $\operatorname{arc}\left(m x_{v}^{-} n\right)$. This requires that $\operatorname{arc}(\bar{m} \bar{n})$ intersects $C_{s}$ in $D_{v}$ which contradicts that $C_{t}$ and $C_{s}$ intersect twice in $\bar{D}_{v}$. We now examine the two cases.

Case $1\left(\bar{m}\right.$ and $\bar{n}$ are on $\left.\operatorname{arc}\left(m x_{v}^{+} n\right)\right)$ : The arc $\operatorname{arc}\left(\bar{m} x_{t}^{-} \bar{n}\right)$ is in $D_{v} \cap D_{s}$. Assume the contrary that $\operatorname{arc}\left(\bar{m} x_{t}^{-} \bar{n}\right)$ is in $D_{v / s}$. Consider a walk on $\operatorname{arc}\left(\bar{m} x_{t}^{-} \bar{n}\right)$ starting at point $x_{t}^{-}$in clockwise direction. Given that $C_{t}$ and $C_{v}$ intersects at $\bar{m}$ and $\bar{n}$ on $\operatorname{arc}\left(m x_{v}^{+} n\right)$, we cannot intersect $\operatorname{arc}\left(m x_{v}^{-} n\right)$ while walking on $C_{t}$ clockwise. Similarly, since $C_{t}$ and $C_{s}$ intersect at $m_{1}$ and $n_{1}$ on $\operatorname{arc}\left(m x_{s}^{+} n\right)$, we cannot intersect $\operatorname{arc}\left(m x_{s}^{-} n\right)$ while walking on $C_{t}$ clockwise. This then makes it impossible to complete the walk as the $\operatorname{arc}\left(\bar{m} x_{t}^{-} \bar{n}\right)$ is assumed to be in $D_{v / s}$. As a result, $\operatorname{arc}\left(\bar{m} x_{t}^{-} \bar{n}\right) \notin D_{v / s}$. Consider a walk on $\operatorname{arc}\left(\bar{m} x_{t}^{-} \bar{n}\right)$ starting at point $x_{t}^{-}$in clockwise direction. Note that we cannot intersect $\operatorname{arc}\left(m x_{s}^{-} n\right)$ but intersect $\operatorname{arc}\left(m x_{v}^{+} n\right)$ at a point $\bar{m}$. In our walk, before we intersect $\bar{n}$, we have to intersect $m_{1}$ and $n_{1}$ as $C_{t}$ has to intersect $C_{s}$ twice on $\operatorname{arc}\left(m x_{s}^{+} n\right)$. Let the first intersection be $m_{1}$ and the second one be $n_{1}$. Finally, we intersect $\bar{n}$ to get back into $D_{v} \cap D_{s}$ and reach back to $x_{t}^{-}$. An example of the resulting coverage scenario for $D_{v}$, $D_{s}$ and $D_{t}$ is given in Figure 3-(c).

Consider the intersection points $m_{1}$ and $n_{1}$ and let $l_{1}$ and $l_{2}$ be two lines that originate at $v$ and cross $m_{1}$ and $n_{1}$ respectively. Observe that $l_{1}$ and $l_{2}$ divide $D_{s / v} \cup D_{t / v}$ into three RCCAs such that part of $D_{s / v} \cup D_{t / v}$ between $l_{2}$ and $l_{1}$ is an RCCA that $t$ is essential to cover and the parts of $D_{s / v} \cup D_{t / v}$ from $n$ to $l_{2}$ and from $l_{1}$ to $m$ are two RCCAs that $s$ is essential to cover w.r.t. $S$.

Case $2\left(\bar{m}\right.$ and $\bar{n}$ are on $\left.\operatorname{arc}\left(m x_{v}^{-} n\right)\right)$ : Given that $s$ is on positive x-axis, $t$ is in $(I I \cup I V)$ as otherwise $C_{t / v}$ and $C_{s / v}$ cannot intersect twice. This requires that $x_{t}^{-} \in D_{v}$. Note that $x_{t}^{-} \notin\left(D_{v} \cap D_{s}\right)$ as otherwise $C_{t}$ intersects either $\operatorname{arc}\left(m x_{s}^{-} n\right)$ or $\operatorname{arc}\left(m x_{v}^{+} n\right)$ contradicting our assumptions that $C_{t}$ intersects $C_{s}$ on $\operatorname{arc}\left(m x_{s}^{+} n\right)$ or that $C_{t}$ intersects $C_{v}$ on $\operatorname{arc}\left(m x_{v}^{-} n\right)$, respectively. A walk on $C_{t}$ starting at $x_{t}^{-}$in clockwise direction first intersects $C_{v}$ at a point $\bar{m}$ as it exits $D_{v}$. It needs to then intersect $C_{s}$ at $m_{1}$ and $n_{1}$ before it enters into $D_{v}$ at a point $\bar{n}$. Assume the contrary that it enters $D_{v}$ at $\bar{n}$ without intersecting $C_{s}$ outside $D_{v}$. This requires that $C_{t}$ intersect $C_{s}$ in $D_{v}$ which is a contradiction. Thus, the sequence of intersections of $C_{t}$ on this walk is $\bar{m}, m_{1}, n_{1}$, and $\bar{n}$. An example of the resulting coverage scenario for $D_{v}, D_{s}$ and $D_{t}$ is given in Figure 3-(d).

Consider the intersection points $m_{1}$ and $n_{1}$ and let $l_{1}$ and $l_{2}$ be two lines that originate at $v$ and cross $m_{1}$ and $n_{1}$ respectively. Observe that $l_{1}$ and $l_{2}$ divide $D_{s / v} \cup D_{t / v}$ into three RCCAs such that part of $D_{s / v} \cup D_{t / v}$ between $l_{2}$ and $l_{1}$ is an RCCA that $s$ is essential to cover and the parts of $D_{s / v} \cup D_{t / v}$ from $n$ to $l_{2}$ and from $l_{1}$ to $m$ are two RCCAs that $t$ is essential to cover w.r.t. $S$.

Lemma 4: Let $S=\{s, t\} \subseteq N(v)$ in an instance of MFSP problem at $v$. If $C_{s / v}$ has two intersections with $C_{t / v}$, then $t \in D_{s}$.

Proof of Lemma 4: Recall that $s$ is on positive x-axis and the two intersection points $m$ and $n$ for $C_{v}$ and $C_{s}$ are on east of $\mathrm{y}$-axis. This implies that $\left|\operatorname{arc}\left(m x_{s}^{+} n\right)\right|$ is in $\left(\pi, \frac{4}{3} \pi\right)$. Given that $C_{t / v}$ intersects $C_{s / v}$ twice at points $m_{1}$ and $n_{1}, \operatorname{arc}\left(m_{1} x_{s}^{+} n_{1}\right)$ is a segment of $\operatorname{arc}\left(m x_{s}^{+} n\right)$ and $\left|\operatorname{arc}\left(m_{1} x_{s}^{+} n_{1}\right)\right| \leq\left|\operatorname{arc}\left(m x_{s}^{+} n\right)\right|$. From Section III-C, when $C_{s}$ and $C_{t}$ intersect twice, we have two cases: (1) $1<|t s|<2$ resulting that $\left|\operatorname{arc}\left(m_{1} x_{s}^{+} n_{1}\right)\right|$ in $\left(\frac{4}{3} \pi, 2 \pi\right)$ and (2) $|t s|<1$ resulting that $\left|\operatorname{arc}\left(m_{1} x_{s}^{+} n_{1}\right)\right|$ in $\left(\frac{2}{3} \pi, \frac{4}{3} \pi\right)$. The case $1<$ $|t s|<2$ is not possible as it requires $\left|\operatorname{arc}\left(m_{1} x_{s}^{+} n_{1}\right)\right|$ to be in $\left(\frac{4}{3} \pi, 2 \pi\right)$ whereas we have $\left|\operatorname{arc}\left(m_{1} x_{s}^{+} n_{1}\right)\right| \leq\left|\operatorname{arc}\left(m x_{s}^{+} n\right)\right|<$ $\frac{4}{3} \pi$. As a result, $|t s|<1$ and $t \in D_{s}$. 


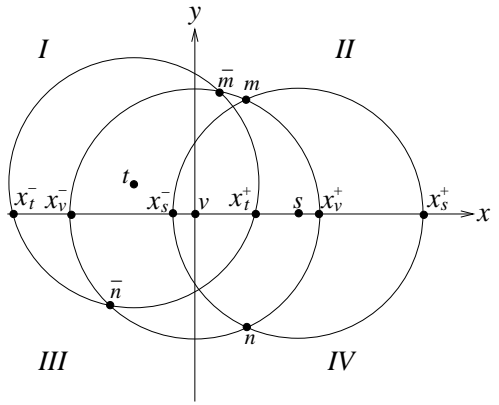

(a)

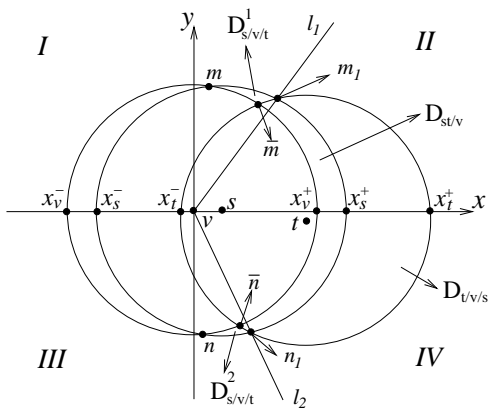

(c)

Fig. 3. Some geometric relations of three intersecting unit disks.

\section{Geometrical Properties}

\section{A. Two-Set Property}

Theorem 1 (Two-Set Property): Given an instance of the MFSP problem (i.e., a node $v$ and its 1-hop and 2-hop neighbor sets $N(v)$ and $N_{2}(v)$ ), the coverage relation presented in Figure 4-(a) is not possible.

Proof of Theorem 1: The scenario in Figure 4-(a) is related to coverage relation between $b_{1}, b_{2}$ and $b_{3}$ all in $N(v)$. In the figure $\left\{a_{1}, a_{2}, \ldots, a_{6}\right\} \in N_{2}(v)$ are a subset of radially ordered 2-hop neighbors of $v$. Observe that, in this setup, $b_{1}$ covers three disjoint MCIs, $M C I_{1}$ including $a_{1}, M C I_{3}$ including $a_{3}$, and $M C I_{5}$ including $a_{5}$. Let $m$ and $n$ be the intersection points between $C_{b_{1}}$ and $C_{v}, m_{1}$ and $n_{1}$ be the ones between $C_{b_{1}}$ and $C_{b_{2}}$, and $m_{2}$ and $n_{2}$ be the ones between $C_{b_{1}}$ and $C_{b_{3}}$. Figure 4-(b) shows an example scenario corresponding to the coverage relation presented in Figure 4(a). In the figure, the lines between $b_{i} \mathrm{~s}$ and $a_{i} \mathrm{~s}$ indicate the coverage of $b_{i}$ on $a_{i}$.

Now, from Lemma $4,\left\{b_{2}, b_{3}\right\} \in N\left(b_{1}\right)$. From the discussion in Section III-C, $|\operatorname{arc}(m n)|<\frac{4}{3} \pi$. Similarly, the coverage relation in Figure 4-(a) requires that $\left|\operatorname{arc}\left(m_{1} n_{1}\right)\right|>\frac{2}{3} \pi$ and $\left|\operatorname{arc}\left(m_{2} n_{2}\right)\right|>\frac{2}{3} \pi$ contradicting $|\operatorname{arc}(m n)|<\frac{4}{3} \pi$. As a result, the coverage relation shown in Figure 4-(a) is not possible.

Assumption 1: Assume that the coverage relation presented in Figure 4-(c) is not possible.

The reason for this assumption is that our algorithm considers the interval of 2-hop nodes in $N_{2}(v)$ (i.e., $\left(a_{i}, n\right)$ ) as a noncircular interval, i.e., it ignores that $a_{i}$ follows $a_{i+n-1}$ in circular order. Note that, considering circularity of the nodes in $N_{2}(v)$, Figure 4-(a) implies that $b_{1}$ cannot be essential

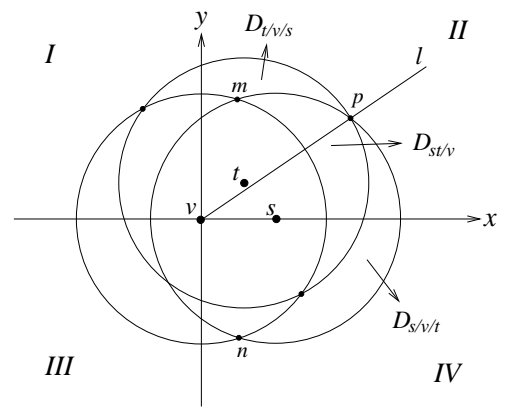

(b)

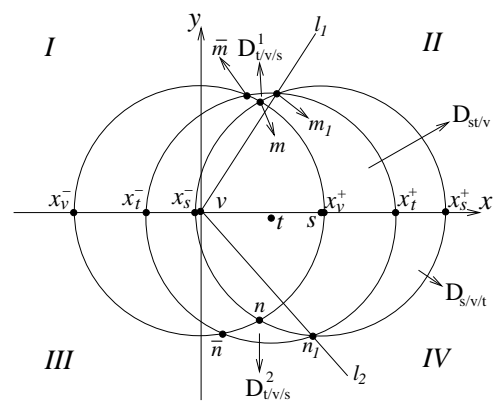

(d)

for more than two MCIs in an optimal solution. Similarly, assuming non-circularity, Figure 4-(c) also implies the same property for $b_{1}$.

\section{B. Non-Interleaving Property}

Definition 9 (Interleaving Coverage): Consider two nodes $\left\{b_{1}, b_{2}\right\} \in N(v)$ in an instance of MFSP problem at $v$. Assume $b_{1}$ covers $\left\{a_{1}, a_{3}\right\} \in N_{2}(v)$ but does not cover $\left\{a_{2}, a_{4}\right\} \in$ $N_{2}(v)$. Similarly, assume $b_{2}$ covers $\left\{a_{2}, a_{4}\right\} \in N_{2}(v)$ but does not cover $\left\{a_{1}, a_{3}\right\} \in N_{2}(v)$. Finally, assume that the radial order between the nodes in $N_{2}(v)$ is as $\left(a_{1}>a_{2}>a_{3}>a_{4}\right)$. The coverage of this form between the nodes $b_{1}$ and $b_{2}$ is called an interleaving coverage.

Theorem 2 (Non-Interleaving Property): In an instance of the MFSP problem (i.e., a node $v$ and its 1-hop and 2-hop neighbor sets $N(v)$ and $N_{2}(v)$ ), no two nodes $\left\{b_{1}, b_{2}\right\} \in N(v)$ can have interleaving coverage, i.e., the connectivity matrix in Figure 4-(d) is not feasible.

Proof of Theorem 2: Note that interleaving is considered between any two nodes $\left\{b_{1}, b_{2}\right\} \in N(v)$. If $C_{b_{1} / v}$ and $C_{b_{2} / v}$ intersect zero times, $b_{1}$ and $b_{2}$ both have two disjoint coverage areas. If $C_{b_{1} / v}$ and $C_{b_{2} / v}$ intersect once, based on Lemma $2, b_{1}$ (and $b_{2}$ ) covers a single RCCA. When this RCCA includes some node $a_{i} \in N_{2}(v)$, then $b_{1}$ (and $b_{2}$ ) has one single MCI (including such node $a_{i}$ ). Finally, when they intersect twice, based on Lemma 3 , one of the nodes, say $b_{1}$, can cover two MCIs and the other node $b_{2}$ can cover one MCI. Note that, as shown in Figure 3-(c), the two intersections radially separate the coverage areas $D_{b_{1} / v / b_{2}}^{1}, D_{b_{2} / v / b_{1}}$, and $D_{b_{1} / v / b_{2}}^{2}$ and no points $q \in D_{b_{2} / v}$ can exist at west of the two areas $D_{b_{1} / v / b_{2}}^{1}$ and $D_{b_{1} / v / b_{2}}^{2}$. 


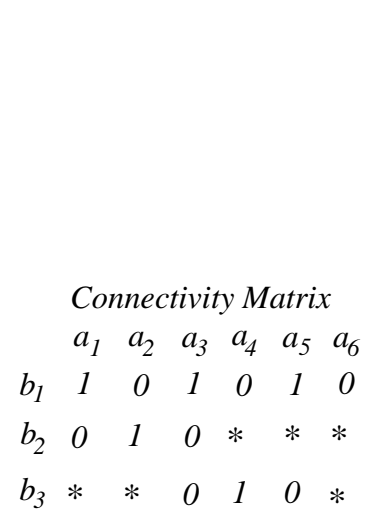

(a)

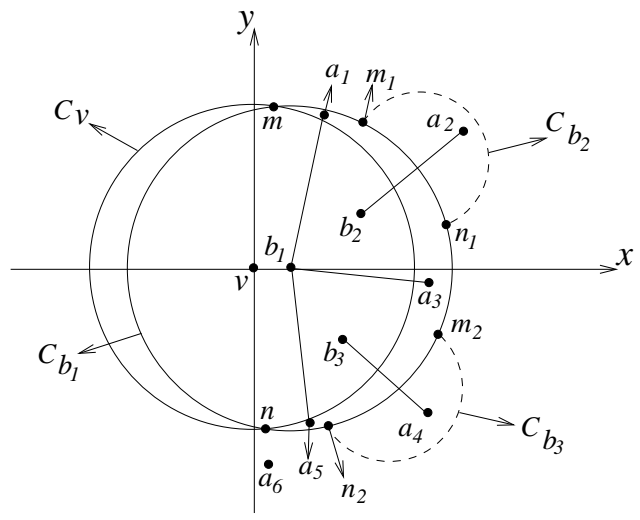

(b)

\begin{tabular}{ccccccc}
\multicolumn{1}{c}{ Connectivity Matrix } \\
& $a_{1}$ & $a_{2}$ & $a_{3}$ & $a_{4}$ & $a_{5}$ \\
$b_{1}$ & 1 & 0 & 1 & 0 & 1 \\
$b_{2}$ & 0 & 0 & 0 & 1 & 0 \\
$b_{3}$ & 0 & 1 & 0 & 0 & 0
\end{tabular}

(c)

\section{Connectivity Matrix $\begin{array}{llll}a_{1} & a_{2} & a_{3} & a_{4}\end{array}$ $\begin{array}{lllll}b_{1} & 1 & 0 & 1 & 0\end{array}$ $\begin{array}{lllll}b_{2} & 0 & 1 & 0 & 1\end{array}$}

(d)

Fig. 4. Unfeasible connectivity scenarios.

\section{Solution to MFSP PROBLEM}

\section{A. Construction}

In this section, we present a dynamic programming algorithm to solve the MFSP problem under unit disk coverage model. The algorithm consists of two parts $A L G 1$ and $A L G 2$ as shown in Figure 5. In an instance of MFSP problem at a node $v$, all 2-hop neighbors of $v$ form a circular interval of length $n=\left|N_{2}(v)\right|$. ALG1 works with non-circular intervals. $A L G 1$ assumes that Assumption 1 and Theorem 2 hold for a given $\left(a_{i}, n\right)$. Hence, the solution of $A L G 1$ for an interval not satisfying Assumption 1 may not be optimal.

Let $S_{\min }\left(a_{i}, j\right)$ be a list of 1-hop neighbors of $v$ that cover the interval $\left(a_{i}, j\right)$. ALG1 uses $N_{\min }\left(a_{i}, n\right)=\left|S_{\min }\left(a_{i}, n\right)\right|$ for ease-of-presentation. Below are the steps of the algorithm in finding a solution for an interval $\left(a_{i}, j\right)$ where $\{i, j\} \in$ $[1, n]$ :

1) Step 1: The best possible solution for $\left(a_{i}, j\right)$ is that the entire interval is covered by a single node $e \in N(v)$. This can be checked by searching the column $f=a_{i}$ of the coverage matrix. If there exists an MCI $C_{e f}=$ $\left(a_{p}, \bar{p}\right)$ that completely includes the interval $\left(a_{i}, j\right)$, then the corresponding one hop neighbor $e$ can be assigned to cover the interval $\left(a_{i}, j\right)$ in the solution. Since this is an assignment with minimum size, i.e., $N_{\min }\left(a_{i}, j\right)=1$, there is no need to check for the other cases below.

2) Step 2: In this step, we split the interval $\left(a_{i}, j\right)$ to two consecutive sub-intervals as $\left(a_{i}, k\right)$ and $\left(a_{i+k}, j-k\right)$. We can combine the optimal solutions of these intervals and this will be a solution to $\left(a_{i}, j\right)$. We consider each possible case for splitting the interval $\left(a_{i}, j\right)$ into two intervals. There are $j-1$ possible cases. Since we are interested in minimum cardinality solution, we take the minimum one in cardinality.

3) Step 3: In this step, we pick a special 1-hop node $s$ which covers $a_{i}$ and $a_{i+j-1}$ (end nodes of $\left(a_{i}, j\right)$ ) and find the MCIs $\left(a_{p}, \bar{p}\right)$ and $\left(a_{q}, \bar{q}\right)$ that $s$ covers such that $a_{i} \in\left(a_{p}, \bar{p}\right)$ and $a_{i+j-1} \in\left(a_{q}, \bar{q}\right)$. A solution in this case can be given by $\{s\} \cup S_{\min }\left(a_{r}, \bar{r}\right)$ where $\left(a_{r}, \bar{r}\right)=\left\{a_{p+\bar{p}}, \ldots, a_{q-1}\right\}$. We find such solutions for all possible $s$ and save it as the optimal solution if it is better than the current solution.

Starting from $j=1, A L G 1$ applies the above procedure for all intervals of lengths up to $j=n$. For an interval $\left(a_{i}, j\right)$, it considers possible solution scenarios by applying the above procedure. Among those solutions, it chooses the one that gives the minimum size solution as $S_{\min }\left(a_{i}, j\right)$. At the end, the algorithm returns a solution as $S_{\min }\left(a_{i}, n\right)$. The running time of the algorithm is $O\left(n^{3}+n^{2} m\right)$ where $O\left(n^{3}\right)$ comes from Step 2 and $O\left(n^{2} m\right)$ comes from Steps 1 and 3. ALG2 calls $A L G 1 n$ times for $\left(a_{i}, n\right)$ with $i=[1, n]$ resulting in the overall complexity $O\left(n^{4}+n^{3} m\right)$. The overall run time complexity can be reduced to $O\left(n^{3}+n^{2} m\right)$ by modifying $A L G 1$ to compute $S_{\min }\left(a_{i}, n\right)$ for all $\left(a_{i}, n\right)$ in one call by changing the line 8 to "FOR $i:=1$ to $\mathrm{n}$ " and by having $A L G 2$ to choose the minimum size $S_{\min }\left(a_{i}, n\right)$ returned by $A L G 1$. The space complexity of the algorithm is $\Theta\left(n^{2} k\right)$ where $k$ represents an upper bound for the number of forwarding nodes in an optimal solution $(k<\min (n, m))$. This bound can be reduced to $\Theta\left(n^{2}\right)$ by saving special indices instead of forwarding node sets for each interval. For each interval, after calculating the optimal solution, we save an index to represent how the optimal solution is found. If the optimal solution is found in Step 1, the 1-hop node covering the whole interval is saved. For Step 2, the optimal split point is saved. For Step 3, 1-hop node and the identity of the middle interval is saved. In this way, the entry for each interval is in constant size and space complexity is $\Theta\left(n^{2}\right)$. In this method interval entries should be traversed back to find the elements of forwarding set. Recall that 2-hop neighborhood information is an input to the algorithm and Calinescu [30] proposes methods to calculate 2-hop neighborhood information with a message complexity of $O(n)$. After the execution of the algorithm, the node $v$ includes the identities of the selected forwarding nodes into the broadcast message. Hence, this step does not incur any additional message overhead.

\section{B. Proof of Correctness: Part 1}

Theorem 3: Given an $\left(a_{i}, j\right)$ where Assumption 1 and Theorem 2 hold, $A L G 1$ finds an optimal solution to $\left(a_{i}, j\right)$ provided that optimal solutions to all continuous subintervals of $\left(a_{i}, j\right)$ are known. 


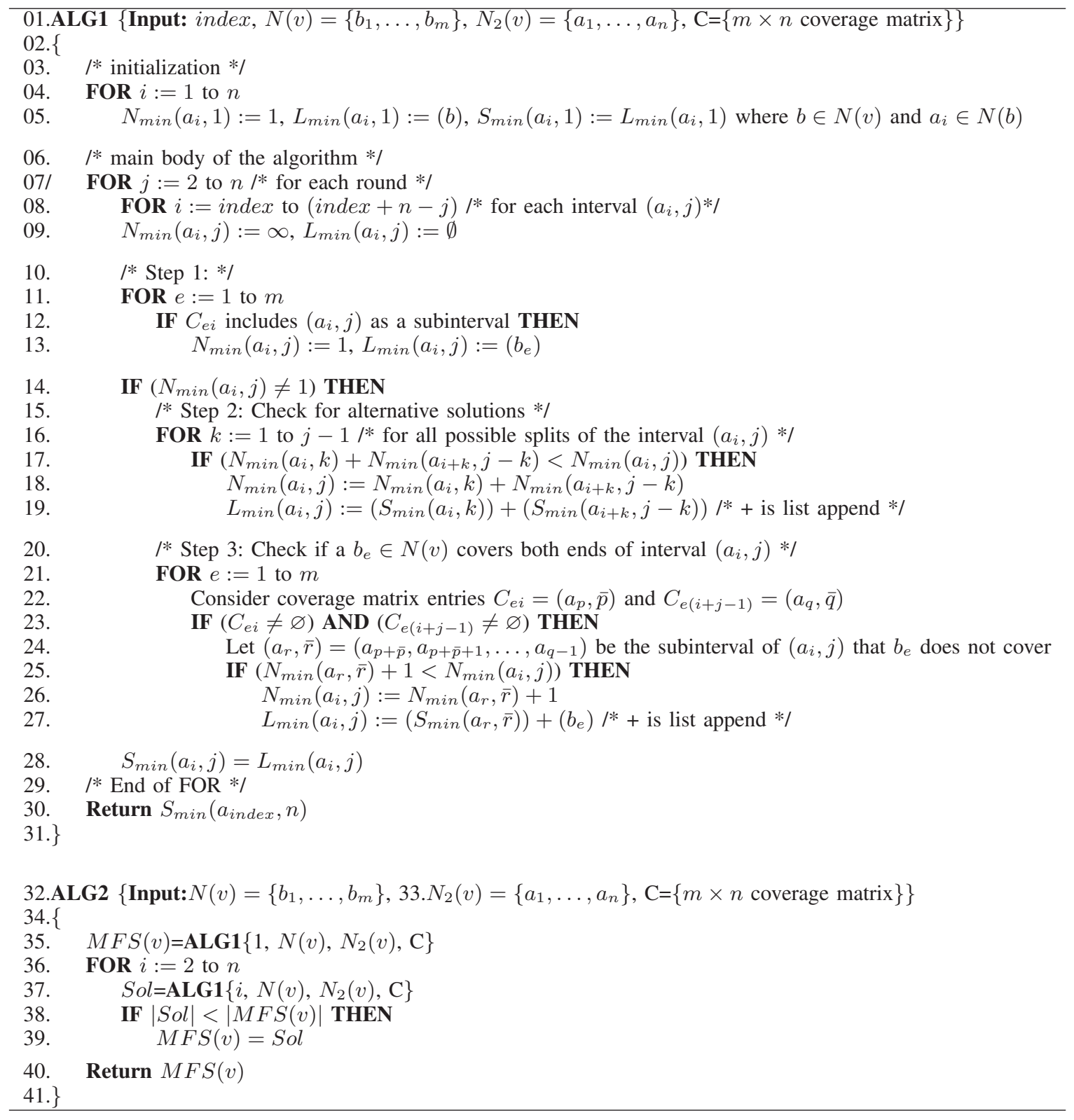

Fig. 5. Outline of the algorithms $A L G 1$ and $A L G 2$.

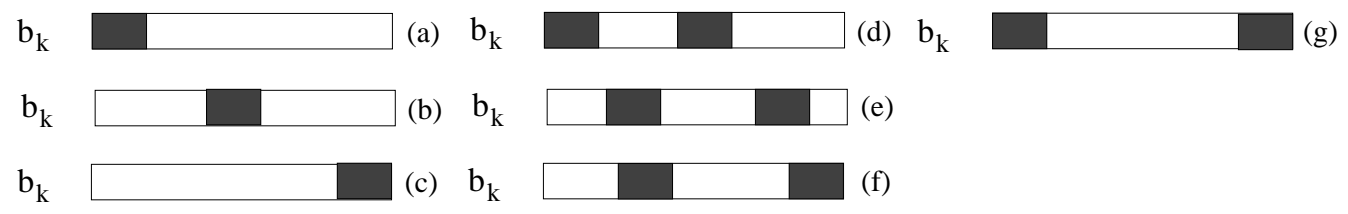

Fig. 6. Possible MCIs $b_{k}$ is essential for in $S$. 
Proof of Theorem 3: Let $S$ be an optimal solution for $\left(a_{i}, j\right)$. Let $b_{k} \in S$ be a node that covers the largest MCI starting from $a_{i}$, i.e., $b_{k}$ covers $\left(a_{i}, \bar{x}\right)$ and for any $b_{l} \neq b_{k} \in S$ covering an MCI $\left(a_{i}, \tilde{x}\right), \tilde{x} \leq \bar{x}$. Note that we know only the existence of such a $b_{k}$ and do not need to know its identity. We analyze the relations between the coverage characteristics of $b_{k}$ and all other nodes in $S$ in all possible coverage cases. Note that $b_{k}$ is essential for at least one and at most two MCIs in $\left(a_{i}, j\right)$. It is essential for at least one MCI because it is in $S$. It is essential for at most two MCIs by Assumption 1. Figure 6 presents possible configurations of MCIs where $b_{k}$ is essential for in $S$. In the following, we first show that the cases in Figure 6-(e),(f) are not possible and then present how our algorithm handles the other cases.

Claim 1: The MCIs that $b_{k}$ is essential for cannot be as in Figure 6-(e),(f).

Proof of Claim 1: Assume that this is not the case and $b_{k}$ is essential for two MCIs $\left(a_{z}, \bar{z}\right)$ and $\left(a_{d}, \bar{d}\right)$ as in Figure 7(a) or -(b). Note that these cases correspond to the cases in Figure 6-(e),(f). Let $b_{l} \in S$ be a node covering $a_{y}$ and $b_{m} \in S$ be a node covering $a_{c}$ in the figure. Note that since $b_{k}$ does not cover either $a_{y}$ or $a_{c}, b_{l} \neq b_{m}$ due to non-interleaving property. Note also that since $b_{k}$ covers the largest MCI $\left(a_{i}, \bar{x}\right)$, $b_{l}$ cannot cover this MCI completely. Finally, since $b_{k}$ is assumed to be essential for $\left(a_{z}, \bar{z}\right)$ and $\left(a_{d}, \bar{d}\right)$, neither $b_{l}$ nor $b_{m}$ can cover these MCIs completely. This then causes a violation of Assumption 1 by $b_{k}$ where the coverage relation between $b_{k}, b_{l}$, and $b_{m}$ is similar to the one in Figure 4-(c).

Case 1: Assume $b_{k}$ is essential for only one MCI on one of the ends of the interval, e.g., $\left(a_{i}, \bar{x}\right)$ as in Figure 7-(a) (or $\left(a_{d}, \bar{d}\right)$ as in Figure 7-(b)). We explain the behavior of the algorithm using the first case and the same argument applies for the second case. Since $b_{k}$ is not essential for any other nodes in $\left(a_{y}, j-\bar{x}\right), S \backslash\left\{b_{k}\right\}$ covers $\left(a_{y}, j-\bar{x}\right)$. Consider an optimal solution $S_{\text {opt }}\left(a_{y}, j-\bar{x}\right)$ for the interval $\left(a_{y}, j-\bar{x}\right)$. Note that $\left|S_{\text {opt }}\left(a_{y}, j-\bar{x}\right)\right|$ cannot be larger than $\left|S \backslash\left\{b_{k}\right\}\right|$ as the former is an optimal solution and the latter is a solution covering $\left(a_{y}, j-\right.$ $\bar{x})$, i.e., $\left|S_{\text {opt }}\left(a_{y}, j-\bar{x}\right)\right| \leq\left|S \backslash\left\{b_{k}\right\}\right|=|S|-1$. Similarly, an optimal solution $S_{\text {opt }}\left(a_{i}, \bar{x}\right)$ for the interval $\left(a_{i}, \bar{x}\right)$ can not be larger than $\left|\left\{b_{k}\right\}\right|$ as the former is an optimal solution and the latter is a solution covering $\left(a_{i}, \bar{x}\right)$. This makes $S_{\text {opt }}\left(a_{i}, \bar{x}\right) \cup$ $S_{\text {opt }}\left(a_{y}, j-\bar{x}\right)$ an optimal solution for $\left(a_{i}, j\right)$ as we assumed that $S$ is an optimal solution for $\left(a_{i}, j\right)$.

This case covers the essential coverage scenarios of $b_{k}$ in $S$ as presented in Figure 6-(a),-(c). If the optimal solution $S$ is of this nature, since we know the optimal solutions for all intervals of length up to $j-1$, the presented algorithm finds this solution in Step 2 by looking at each split points for the interval $\left(a_{i}, j\right)$.

Case 2: Assume that $b_{k}$ is essential for two MCIs $\left(a_{i}, \bar{x}\right)$ and $\left(a_{d}, \bar{d}\right)$ at both ends of $\left(a_{i}, j\right)$ as shown in Figure 7-(b). In this case, $S \backslash\left\{b_{k}\right\}$ covers $\left(a_{y}, j-(\bar{x}+\bar{d})\right)$ since $b_{k}$ is not essential for that part. Consider an optimal solution $S_{\text {opt }}\left(a_{y}, j-(\bar{x}+\bar{d})\right)$ for the interval $\left(a_{y}, j-(\bar{x}+\bar{d})\right)$. Note that $\left|S_{o p t}\left(a_{y}, j-(\bar{x}+\bar{d})\right)\right|$ cannot be larger than $\left|S \backslash\left\{b_{k}\right\}\right|$ as the former is an optimal solution and the latter is a solution $\left(a_{y}, j-(\bar{x}+\bar{d})\right)$, i.e., $\left|S_{\text {opt }}\left(a_{y}, j-(\bar{x}+\bar{d})\right)\right| \leq\left|S \backslash\left\{b_{k}\right\}\right|=|S|-1$. This then requires that $\left\{b_{k}\right\} \cup S_{o p t}\left(a_{y}, j-(\bar{x}+\bar{d})\right)$ is an optimal solution for $\left(a_{i}, j\right)$ as we started with an assumption that $S$ is an optimal solution for $\left(a_{i}, j\right)$, i.e., $\left|S_{\text {opt }}\left(a_{y}, j-(\bar{x}+\bar{d})\right)\right|+\left|\left\{b_{k}\right\}\right| \leq|S|$.

This case covers the essential coverage scenarios of $b_{k}$ in $S$ as presented in Figure 6-(g). If the optimal solution $S$ is of this nature, since we know the optimal solutions for all intervals of length up to $j-1$, the presented algorithm finds this solution in Step 3.

Case 3: Assume that $b_{k}$ is essential for two MCIs $\left(a_{i}, \bar{x}\right)$ and $\left(a_{z}, \bar{z}\right)$ as shown in Figure 7-(a) or -(b). In this scenario, we divide the nodes in $S$ into two subgroups, $S_{1}$ and $S_{2}$ in a way that $S_{1} \cup S_{2}=S$ and $S_{1} \cap S_{2}=\varnothing$. Our goal is to create $S_{1}$ and $S_{2}$ such that $S_{1}$ covers $\left(a_{i}, \bar{x}+\bar{y}+\bar{z}\right)$ and $S_{2}$ covers $\left(a_{c}, j-(\bar{x}+\bar{y}+\bar{z})\right)$. When we assign a node $b_{x}$ to $S_{1}$ (or $S_{2}$ ), if we can guarantee that there exist another node $b_{y}$ in $S_{2}$ (or $S_{1}$ ) which covers all the nodes that $b_{x}$ covers in $\left(a_{c}, j-(\bar{x}+\bar{y}+\bar{z})\right)\left(\right.$ or $\left.\left(a_{i}, \bar{x}+\bar{y}+\bar{z}\right)\right)$, then this partition will give two sets $S_{1}$ and $S_{2}$ with the desired properties. Note that $b_{k}$ is the only node covering $\left(a_{i}, \bar{x}\right)$ in $S$ as in Figure 7-(a) or -(b). Then, there is a node, say $b_{m}$, in $S$ which covers $a_{c}$.

Claim 2: $b_{k}$ should cover all nodes that $b_{m}$ covers in $\left(a_{i}, \bar{x}+\bar{y}+\bar{z}\right)$.

Proof of Claim 2: $b_{k}$ covers a node in $\left(a_{i}, \bar{x}\right)$ and a node in $\left(a_{z}, \bar{z}\right)$ that are not covered by $b_{m} . b_{m}$ covers $a_{c}$ that is not covered by $b_{k}$. If $b_{k}$ does not cover a node that $b_{m}$ covers in $\left(a_{y}, \bar{y}\right)$, this leads to an interleaving coverage between $b_{k}$ and $b_{m}$ contradicting Theorem 2 .

Claim 3: $b_{m}$ should cover all the nodes that $b_{k}$ covers in $\left(a_{c}, j-(\bar{x}+\bar{y}+\bar{z})\right)$.

Proof of Claim 3: Recall that $b_{k}$ does not cover $a_{y}$. Let $b_{l}$ be a node covering $a_{y}$. Note that $b_{l} \neq b_{m}$ as otherwise $b_{k}$ and $b_{l}$ have an interleaving coverage. Now, $b_{k}$ covers nodes in $\left(a_{i}, \bar{x}\right)$ and in $\left(a_{z}, \bar{z}\right)$ which $b_{l}$ does not cover. Note also that $b_{m}$ covers $a_{c}$ which is not covered by $b_{k}$. If $b_{m}$ does not cover a node $a_{d} \in\left(a_{c}, j-(\bar{x}+\bar{y}+\bar{z})\right)\left(a_{d} \neq a_{c}\right)$ that $b_{k}$ covers, then this causes a violation of Assumption 1 where the coverage relation between $b_{k}, b_{l}$, and $b_{m}$ is similar to the one in Figure 4-(c). Thus, $b_{m}$ covers all such $a_{d} \in\left(a_{c}, j-(\bar{x}+\bar{y}+\bar{z})\right)$.

Based on Claims 2 and 3, we can put $b_{k}$ into $S_{1}$ and $b_{m}$ into $S_{2}$. For any other node $b_{o}$ :

1) If $b_{o}$ covers a node in $\left(a_{c}, j-(\bar{x}+\bar{y}+\bar{z})\right)$ which is not covered by $b_{k}$, then $b_{o} \in S_{2}$ and $b_{k}$ covers all nodes that $b_{o}$ covers in $\left(a_{i}, \bar{x}+\bar{y}+\bar{z}\right)$ due to Theorem 2 .

2) If $b_{o}$ covers a node in $\left(a_{y}, \bar{y}\right)$ which is not covered by $b_{k}$, then $b_{o} \in S_{1}$ and $b_{k}$ covers all nodes that $b_{o}$ covers in $\left(a_{c}, j-(\bar{x}+\bar{y}+\bar{z})\right)$ due to Theorem 2. From Claim 3, $b_{m}$ covers all nodes that $b_{k}$ covers in $\left(a_{c}, j-(\bar{x}+\bar{y}+\right.$ $\bar{z}))$. Therefore, $b_{m}$ covers all nodes that $b_{o}$ covers in $\left(a_{c}, j-(\bar{x}+\bar{y}+\bar{z})\right)$.

Note that $b_{o}$ should be in one of the above two cases as otherwise $b_{o} \notin S$. Based on the above construction, $S_{1}$ covers $\left(a_{i}, \bar{x}+\bar{y}+\bar{z}\right)$ and $S_{2}$ covers $\left(a_{c}, j-(\bar{x}+\bar{y}+\bar{z})\right)$. Consider optimal solutions $S_{\text {opt }}\left(a_{i}, \bar{x}+\bar{y}+\bar{z}\right)$ and $S_{\text {opt }}\left(a_{c}, j-(\bar{x}+\bar{y}+\right.$ $\bar{z}))$. We have $\left|S_{\text {opt }}\left(a_{i}, \bar{x}+\bar{y}+\bar{z}\right)\right| \leq\left|S_{1}\right|$ and $\mid S_{\text {opt }}\left(a_{c}, j-\right.$ $(\bar{x}+\bar{y}+\bar{z}))|\leq| S_{2} \mid$. Since $S=S_{1} \cup \bar{S}_{2}$ is an optimal solution, by Step 2 of $A L G 1$, we have $\left|L_{\min }\left(a_{i}, j\right)\right| \leq \mid S_{\text {opt }}\left(a_{i}, \bar{x}+\right.$ $\bar{y}+\bar{z})|+| S_{\text {opt }}\left(a_{c}, j-(\bar{x}+\bar{y}+\bar{z})\right)|\leq| S_{1}|+| S_{2}|=| S \mid$. 


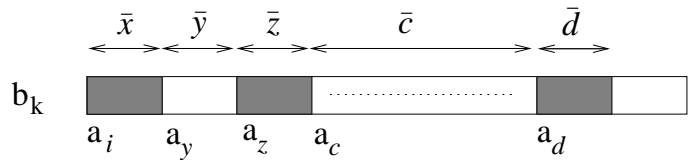

(a)

Fig. 7. Coverage characteristics of $b_{k}$ in $S$.

This case covers the essential coverage scenarios of $b_{k}$ in $S$ as presented in Figure 6-(d). If the optimal solution $S$ is of this nature, since we know the optimal solutions for all intervals of length up to $j-1$, the presented algorithm finds this solution in Step 2.

Case 4: We now examine the case where $b_{k}$ is essential for one interval (e.g., $\left(a_{z}, \bar{z}\right)$ in Figure 7$)$ which corresponds to the case in Figure 6-(b). Similar to the above discussion, we again divide $S$ into $S_{1}$ and $S_{2}$ and try to put nodes $b_{x}$ into one of these two sets such that $S_{1} \cup S_{2}=S$ and $S_{1} \cap S_{2}=\varnothing$. Let $b_{m} \in S$ be a node covering $a_{c}$ and $b_{l} \in S$ be a node covering $a_{y}$ as in Figure 7 . Note that $b_{l}$ cannot cover $\left(a_{i}, \bar{x}\right)$ as otherwise $b_{k}$ would not be covering the largest interval including $a_{i}$. Note also that $b_{l} \neq b_{m}$ as otherwise $b_{k}$ and $b_{l}$ would have an interleaving coverage.

Claim 4: We claim that $b_{k}$ or $b_{l}$ should cover all nodes that $b_{m}$ covers in $\left(a_{i}, \bar{x}+\bar{y}+\bar{z}\right)$.

Proof of Claim 4: Note that if $b_{m}$ does not cover $\left(a_{i}, \bar{x}\right)$, then the proof is the same as the proof of Claim 2 above. If $b_{m}$ covers $\left(a_{i}, \bar{x}\right)$, it can cover some node in $\left(a_{y}, \bar{y}\right)$ and some node in $\left(a_{c}, \bar{c}\right)$ which are not covered by $b_{k}$. If this is the case, $b_{l}$ should cover all the nodes $b_{m}$ covers in $\left(a_{y}, \bar{y}\right)$ as otherwise $b_{m}$ violates Assumption 1. That is, $b_{m}$ covers $\left(a_{i}, \bar{x}\right)$ and some nodes in $a_{\tilde{y}} \neq a_{y} \in\left(a_{y}, \bar{y}\right)$ which $b_{l}$ does not cover; and $b_{l}$ covers $a_{y}$ which $b_{m}$ does not cover. Based on this, $b_{l}$ separates the coverage of $b_{m}$ into two sets. In addition, $b_{m}$ covers $a_{\tilde{y}} \neq a_{y} \in\left(a_{y}, \bar{y}\right)$ and $a_{c}$ which are not covered by $b_{k}$ and $b_{k}$ covers $\left(a_{z}, \bar{z}\right)$ which $b_{m}$ does not cover. The coverage scenario among the three nodes is similar to the case in Figure 4-(c) with $b_{m}, b_{l}$, and $b_{k}$ in the position of $b_{1}, b_{2}$, and $b_{3}$ in the figure, respectively.

Claim 5: $b_{m}$ covers all nodes $b_{k}$ covers in $\left(a_{c}, j-(\bar{x}+\bar{y}+\right.$ $\bar{z}))$.

Proof of Claim 5: $b_{k}$ covers a node in $\left(a_{i}, \bar{x}\right)$ and a node in $\left(a_{z}, \bar{z}\right)$ which are not covered by $b_{l} . b_{l}$ covers $a_{y}$ which is not covered by $b_{k}$. $b_{m}$ covers $a_{c}$ which is not covered by $b_{k}$. If $b_{m}$ does not cover a node that $b_{k}$ covers in $\left(a_{c}, j-\right.$ $(\bar{x}+\bar{y}+\bar{z}))$, this will be a violation of Assumption 1 with a coverage scenario among $b_{k}, b_{l}$, and $b_{m}$ similar to the case in Figure 4-(c) with $b_{k}, b_{l}$, and $b_{m}$ in the position of $b_{1}, b_{2}$, and $b_{3}$ in the figure, respectively.

Claim 6: $b_{k}$ covers all nodes that $b_{l}$ covers in $\left(a_{c}, j-(\bar{x}+\right.$ $\bar{y}+\bar{z}))$.

Proof of Claim $6: b_{k}$ covers nodes in $\left(a_{i}, \bar{x}\right)$ and in $\left(a_{z}, \bar{z}\right)$ which are not covered by $b_{l} . b_{l}$ covers $a_{y}$ that $b_{k}$ does not cover. If $b_{k}$ does not cover a node $b_{l}$ covers in $\left(a_{c}, j-(\bar{x}+\bar{y}+\bar{z})\right)$, this will lead to an interleaving coverage between $b_{k}$ and $b_{l}$ contradicting Theorem 2 .

Combining Claims 5 and $6, b_{m}$ covers all nodes that $b_{l}$ covers in $\left(a_{c}, j-(\bar{x}+\bar{y}+\bar{z})\right)$. Based on these results, we can put $b_{k}$ and $b_{l}$ into $S_{1}$ and $b_{m}$ into $S_{2}$. For any other node $b_{o}$ :

1) If $b_{o}$ does not cover a node in $\left(a_{y}, \bar{y}\right)$ which is not covered by $b_{k}$, then $b_{o} \in S_{2}$.

2) If $b_{o}$ does not cover a node in $\left(a_{c}, j-(\bar{x}+\bar{y}+\bar{z})\right)$ which is not covered by $b_{k}$, then $b_{o} \in S_{1} . b_{m}$ covers all nodes $b_{o}$ covers in $\left(a_{c}, j-(\bar{x}+\bar{y}+\bar{z})\right)$.

3) If $b_{o}$ covers nodes from both $\left(a_{y}, \bar{y}\right)$ and $\left(a_{c}, j-(\bar{x}+\bar{y}+\right.$ $\bar{z}))$ which are not covered by $b_{k}$, then $b_{o} \in S_{2}$. In this case, $b_{l}$ covers all nodes $b_{o}$ covers in $\left(a_{y}, \bar{y}\right)$. Now, to have a non-interleaving coverage between $b_{o}$ and $b_{k}, b_{o}$ should cover $\left(a_{i}, \bar{x}\right)$ as $b_{k}$ covers a node in $\left(a_{z}, \bar{z}\right)$ that $b_{o}$ does not cover. Next, if $b_{l}$ does not cover all nodes $b_{o}$ covers in $\left(a_{y}, \bar{y}\right), b_{o}$ will violate Assumption 1. This case is similar to the case of $b_{m}$ in the proof of Claim 4 and is therefore omitted.

Note that $b_{o}$ should be in one of the above two cases as otherwise $b_{o} \notin S$. Similar to the discussion above, for optimal solutions $S_{o p t}\left(a_{i}, \bar{x}+\bar{y}+\bar{z}\right)$ and $S_{o p t}\left(a_{c}, j-(\bar{x}+\bar{y}+\bar{z})\right)$, we have $\left|S_{\text {opt }}\left(a_{i}, \bar{x}+\bar{y}+\bar{z}\right)\right| \leq\left|S_{1}\right|$ and $\mid S_{\text {opt }}\left(a_{c}, j-(\bar{x}+\right.$ $\bar{y}+\bar{z}))|\leq| S_{2} \mid$. Since $S=S_{1} \cup S_{2}$ is an optimal solution, by Step 2 of $A L G 1$, we have $\left|L_{\text {min }}\left(a_{i}, j\right)\right| \leq \mid S_{\text {opt }}\left(a_{i}, \bar{x}+\bar{y}+\right.$ $\bar{z})|+| S_{\text {opt }}\left(a_{c}, j-(\bar{x}+\bar{y}+\bar{z})\right)|\leq| S_{1}|+| S_{2}|=| S \mid$.

This case covers the essential coverage scenarios of $b_{k}$ in $S$ as presented in Figure 6-(b). If the optimal solution $S$ is of this nature, since we know the optimal solutions for all intervals of length up to $j-1$, the presented algorithm finds this solution in Step 2.

As a result, depending on the nature of the optimal solution for $\left(a_{i}, j\right)$, one of the above mentioned four cases correspond to the optimal solution $S_{\text {opt }}\left(a_{i}, j\right)$. This concludes the proof of Theorem 3.

Lemma 5: ALG1 finds an optimal solution for $\left(a_{i}, j\right)$ if Assumption 1 holds for $\left(a_{i}, j\right)$ and its all continuous subintervals whose solutions contribute to calculate the solution for $\left(a_{i}, j\right)^{1}$.

Proof of Lemma 5: The proof is by induction on $j$. For $j=1$, the optimal solution is found in Step 1 of $A L G 1$. For the hypothesis case, we assume that we have the optimal solution for any $\left(a_{k}, j^{\prime}\right)$ for $j^{\prime}<j$ and $k \in\left[i, i+j^{\prime}-1\right]$ where $\left(a_{k}, j^{\prime}\right)$ contributes to the solution for $\left(a_{i}, j\right)$. By Theorem 3 and the induction hypothesis, since Assumption 1 holds for $\left(a_{i}, j\right)$ and we have the solutions for all contributing subintervals of $\left(a_{i}, j\right), A L G 1$ finds an optimal solution for $\left(a_{i}, j\right)$.

${ }^{1}$ A solution of a subinterval contributes to the solution of $\left(a_{i}, j\right)$ if it is used as part of the selected solution for $\left(a_{i}, j\right)$. As an example, in Figure 8, the solution for $\left(a_{1}, 4\right)$ and $\left(a_{5}, 1\right)$ contribute to the solution of $\left(a_{1}, 5\right)$. 


\section{Proof of Correctness: Part 2}

The example in Figure 8 shows that Assumption 1 may not hold for all $\left(a_{i}, n\right)$. In the example, consider the interval $\left(a_{2}, 5\right)$. In this interval, $s$ is essential to cover three MCIs namely $\left(a_{2}, 1\right),\left(a_{4}, 1\right)$, and $\left(a_{1}, 1\right)$. The problem in this case is that the interval $\left(a_{2}, 5\right)$ starts at the middle of an MCI that $s$ is essential for. Assumption 1 does not hold in this case and the solution returned by $A L G 1$ is not optimal. On the other hand, please note that for another interval, $\left(a_{1}, 5\right)$, Assumption 1 is satisfied.

Theorem 4: Given an instance of the MFSP problem (i.e., a node $v$ and its 1-hop and 2-hop neighbor sets $N(v)$ and $N_{2}(v)$ ), there exists at least one interval $\left(a_{i}, n\right)$ where $i \in$ $[1, n]$ such that $A L G 1$ finds an optimal solution for $\left(a_{i}, n\right)$.

Proof of Theorem 4: Let $S$ be an optimal solution for an instance of MFSP problem. Let $b_{k} \in S$ be essential for an MCI $\left(a_{i}, \bar{x}\right)$. In this case, we represent the nodes in $N_{2}(v)$ as an interval $\left(a_{i}, n\right)$. By the definition of an MCI, since $b_{k}$ is essential for an MCI including $a_{i}, b_{k}$ cannot cover $a_{i+n-1}$, i.e., the node prior to $a_{i}$ in circular order. From Theorem 1, $b_{k} \in S$ may be essential for another MCI $\left(a_{z}, \bar{z}\right)$ in $\left(a_{i}, n\right)$.

Case 1: $\left(b_{k}\right.$ is essential for $\left(a_{i}, \bar{x}\right)$ only). This case is similar to Case 1 in the previous section. That is, $\left(a_{i}, n\right)$ is divided into two intervals $\left(a_{i}, \bar{x}\right)$ and $\left(a_{y}, n-\bar{x}\right)$ where $a_{y}=a_{i+\bar{x}}$. Following the same arguments as in Case 1 in the previous section, we have $S_{o p t}\left(a_{i}, n\right)=S_{o p t}\left(a_{i}, \bar{x}\right) \cup S_{o p t}\left(a_{y}, n-\bar{x}\right)$. $A L G 1$ finds an optimal solution for $\left(a_{i}, \bar{x}\right)$ in Step 1 as $b_{k}$ covers $\left(a_{i}, \bar{x}\right)$. ALG1 finds an optimal solution for $\left(a_{y}, n-\bar{x}\right)$ if Assumption 1 holds for $\left(a_{y}, n-\bar{x}\right)$ and its all continuous subintervals whose solution contribute to the solution of $\left(a_{y}, n-\bar{x}\right)$ by Lemma 5. Assume Assumption 1 does not hold for $\left(a_{y}, n-\bar{x}\right)$ or any of its continuous subintervals whose solution contribute to the solution of $\left(a_{y}, n-\bar{x}\right)$. Let us call the (sub)interval that violates Assumption $1\left(a_{c}, \bar{c}\right)$. There should be a node $b_{m}$ that violates Assumption 1 for $\left(a_{c}, \bar{c}\right)$ similar to $b_{1}$ in Figure 4-(c). In this case, $b_{m}$ should cover all the nodes in $\left(a_{i}, n\right) \backslash\left(a_{c}, \bar{c}\right)$ as otherwise $b_{m}$ would violate Theorem 1 in $\left(a_{i}, n\right)$. If $b_{m} \notin S_{o p t}\left(a_{c}, \bar{c}\right)$, then it can be discarded. If $b_{m} \in S_{\text {opt }}\left(a_{c}, \bar{c}\right)$, then $S_{\text {opt }}\left(a_{i}, n\right)=S_{\text {opt }}\left(a_{y}, n-\bar{x}\right)$ which contradicts that $S_{\text {opt }}\left(a_{i}, n\right)=S_{\text {opt }}\left(a_{i}, \bar{x}\right) \cup S_{\text {opt }}\left(a_{y}, n-\bar{x}\right)$. As a result, no $b_{m}$ can violate Assumption 1 for any $\left(a_{c}, \bar{c}\right)$. Since $A L G 1$ finds optimal solutions $S_{o p t}\left(a_{i}, \bar{x}\right)$ and $S_{\text {opt }}\left(a_{y}, n-\bar{x}\right)$, and $S_{o p t}\left(a_{i}, n\right)=S_{o p t}\left(a_{i}, \bar{x}\right) \cup S_{o p t}\left(a_{y}, n-\bar{x}\right)$ holds, $A L G 1$ finds optimal solution for $\left(a_{i}, n\right)$ in Step 2.

Case 2: $\left(b_{k}\right.$ is essential for $\left(a_{i}, \bar{x}\right)$ and $\left(a_{z}, \bar{z}\right)$.) This case is similar to Case 3 in the previous section. That is, $\left(a_{i}, n\right)$ is divided into two intervals $\left(a_{i}, \bar{x}+\bar{y}+\bar{z}\right)$ and $\left(a_{c}, n-(\bar{x}+\bar{y}+\bar{z})\right)$

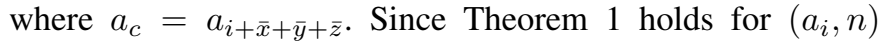
and $b_{k}$ does not cover $a_{i+n-1}$, we can use the arguments in Case 3 in the previous section for this case again. From those arguments, we have $S_{o p t}\left(a_{i}, n\right)=S_{\text {opt }}\left(a_{i}, \bar{x}+\bar{y}+\bar{z}\right) \cup$ $S_{\text {opt }}\left(a_{c}, n-(\bar{x}+\bar{y}+\bar{z})\right)$. ALG1 finds an optimal solution for $\left(a_{i}, \bar{x}+\bar{y}+\bar{z}\right)$ if Assumption 1 holds for $\left(a_{i}, \bar{x}+\bar{y}+\bar{z}\right)$ and its all continuous subintervals whose solution contribute to the solution of $\left(a_{i}, \bar{x}+\bar{y}+\bar{z}\right)$ by Lemma 5 . Assume Assumption 1 does not hold for $\left(a_{i}, \bar{x}+\bar{y}+\bar{z}\right)$ or any of its continuous subintervals whose solution contribute to its solution. Let us call this (sub)interval $\left(a_{p}, \bar{p}\right)$. There should be a node $b_{m}$ that violates Assumption 1 for $\left(a_{p}, \bar{p}\right)$ similar to $b_{1}$ in Figure 4-(c). In this case, $b_{m}$ should cover all the nodes in $\left(a_{i}, n\right) \backslash\left(a_{p}, \bar{p}\right)$ as otherwise $b_{m}$ would violate Theorem 1 in $\left(a_{i}, n\right)$. If $b_{m} \notin$ $S_{\text {opt }}\left(a_{p}, \bar{p}\right)$, then it can be discarded. If $b_{m} \in S_{\text {opt }}\left(a_{p}, \bar{p}\right)$, then $S_{\text {opt }}\left(a_{i}, n\right)=S_{\text {opt }}\left(a_{i}, \bar{x}+\bar{y}+\bar{z}\right)$ which contradicts that $S_{\text {opt }}\left(a_{i}, n\right)=S_{\text {opt }}\left(a_{i}, \bar{x}+\bar{y}+\bar{z}\right) \cup S_{\text {opt }}\left(a_{c}, n-(\bar{x}+\bar{y}+\bar{z})\right)$. As a result, no such $b_{m}$ can violate Assumption 1 in any $\left(a_{p}, \bar{p}\right)$. $A L G 1$ finds an optimal solution for $\left(a_{c}, n-(\bar{x}+\bar{y}+\bar{z})\right)$ with a similar argument. Since $A L G 1$ finds optimal solutions $S_{o p t}\left(a_{i}, \bar{x}+\bar{y}+\bar{z}\right)$ and $S_{o p t}\left(a_{y}, n-(\bar{x}+\bar{y}+\bar{z})\right)$, and $S_{\text {opt }}\left(a_{i}, n\right)=S_{\text {opt }}\left(a_{i}, \bar{x}+\bar{y}+\bar{z}\right) \cup S_{\text {opt }}\left(a_{y}, n-(\bar{x}+\bar{y}+\bar{z})\right)$ holds, $A L G 1$ finds optimal solution for $\left(a_{i}, n\right)$ in Step 2.

Finally, since $A L G 1$ finds the optimal solution for at least one $\left(a_{i}, n\right), A L G 2$ returns this solution by choosing the minimum size solution returned by $A L G 1$.

\section{CONCLUSIONS}

In this paper, we have studied the minimum forwarding set problem (MFSP) in the context of wireless ad hoc networks. Leveraging the practical characteristics of the application environment, we have proposed a polynomial time algorithm to build an optimal solution to the MFSP problem under the unit disk coverage model for wireless transmission. We expect the work presented in this paper to have an impact on the design and development of new algorithms for several wireless network applications including energy efficient multicast and broadcast protocols; energy efficient topology control protocols; and energy efficient virtual backbone construction protocols for wireless ad hoc networks and sensor networks.

\section{REFERENCES}

[1] C. Perkins and E. Royer, "Ad hoc on-demand distance vector routing," in Proceedings of the 2nd IEEE Workshop on Mobile Computing Systems and Applications, (New Orleans, LA, USA), February 1999.

[2] D. Johnson, D. Maltz, and J. Broch, Ad Hoc Networking, ch. Chapter 5: DSR: The Dynamic Source Routing Protocol for Multi-Hop Wireless Ad Hoc Networks, pp. 139-172. Addison-Wesley, 2001.

[3] T. Clausen and P. Jacquet, "Optimized link state routing protocol (OLSR)." Internet Engineering Task Force (IETF) - RFC 3561, July 2003.

[4] M. Thoppian and R. Prakash, "A distributed protocol for dynamic address assignment in mobile ad hoc networks," IEEE Tran. on Mobile Computing, vol. 5, pp. 4-19, January 2006.

[5] S.-Y. Ni, Y.-C. Tseng, Y.-S. Chen, and J.-P. Sheu, "The broadcast storm problem in a mobile ad hoc network," in Proceedings of the 5th annual ACM/IEEE international conference on Mobile computing and networking, (Seattle, WA, USA), August 1999.

[6] F. Ingelrest, D. Simplot-Ryl, and I. Stojmenovic, Resource Management in Wireless Networking, ch. Energy-efficient broadcasting in wireless mobile ad hoc networks. Kluwer Academic Press, 2004.

[7] A. Qayyum, L. Viennot, and A. Laouiti, "Multipoint relaying for flooding broadcast messages in mobile wireless networks," in Proceedings of 35th Hawaii International Conference on System Sciences, (Big Island, HI, USA), January 2002.

[8] G. Calinescu, I. Mandoui, P.-J. Wan, and A. Zelikovsky, "Selecting forwarding neighbors in wireless ad hoc networks," Mobile Networks and Applications, vol. 9, pp. 101-111, 2004.

[9] V. Chvatal, "A greedy heuristic for the set-covering problem," Mathematics of Operation Research, vol. 4, no. 3, pp. 233-235, 1979.

[10] A. Busson, N. Mitton, and E. Fleury, "An analysis of the mpr selection in olsr and consequences," in Mediterranean Ad Hoc Networking Workshop (MedHocNet), (Ile de Porquerolles, France), June 2005.

[11] B. Clark, C. Colbourn, and D. Johnson, "Unit disk graphs," Discrete Mathematics, vol. 86, pp. 165-177, 1990. 


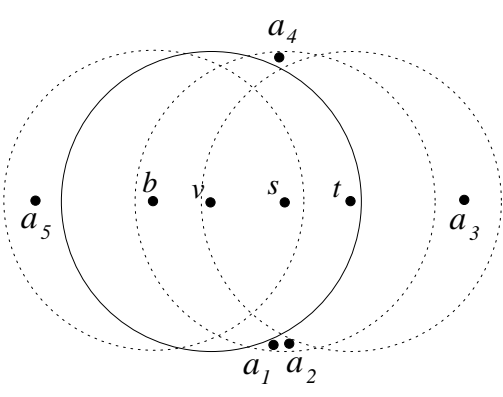

(a)

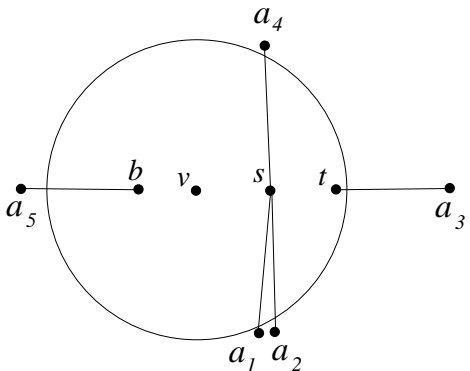

(b)

Fig. 8. An example on finalization.

[12] J. Wu, W. Lou, and F. Dai, "Extended multipoint relays to determine connected dominating sets in MANETs," IEEE Transactions on Computers, vol. 55, pp. 334-347, March 2006.

[13] X.-Y. Li and I. Stojmenovic, Handbook of Algorithms for Mobile and Wireless Networking and Computing, ch. Broadcasting and Topology Control in Wireless Ad Hoc Networks. CRC Press, November 2005.

[14] J. Wu and W. Lou, "Forward-node-set-based broadcast in clustered mobile ad hoc networks," Wireless Communications and Mobile Computing, vol. 3, no. 2, pp. 155-173, 2003.

[15] B. Williams and T. Camp, "Comparison of broadcasting techniques for mobile ad hoc networks," in Proceedings of MOBIHOC, (Lausanne, Switzerland), June 2002.

[16] D. S. Johnson, "Approximation algorithms for combinatorial problems," Journal of Computer and System Sciences, vol. 9, pp. 256-278, 1974.

[17] L. Lovasz, "On the ratio of optimal integral and fractional covers," Discrete Mathematics, vol. 13, no. 4, pp. 383-390, 1975.

[18] D. Hochbaum, "Approximation Algorithms for the Set Covering and Vertex Cover Problems," SIAM J. Comput., vol. 11, no. 3, pp. 555-556, 1982.

[19] R. Bar-Yehuda and S. Even, "A Linear-Time Approximation Algorithm for the Weighted Vertex Cover Problem," J. Algorithms, vol. 2, no. 2, pp. 198-203, 1981.

[20] V. Paschos, "A survey of approximately optimal solutions to some covering and packing problems," ACM Computing Surveys (CSUR), vol. 29, no. 2, pp. 171-209, 1997.

[21] M. Marathe, H. Breu, H. Hunt, S. Ravi, and D. Rosenkrantz, "Simple heuristics for unit disk graphs," Networks, vol. 25, pp. 59-68, 1995.

[22] H. Hunt, M. Marathe, V. Radhakrishnan, S. Ravi, D. Rosenkrantz, and R. Stearns, "Nc-approximation schemes for np-and pspace-hard problems for geometric graphs," Journal of Algorithms, vol. 26, no. 2, pp. 238-274, 1998.

[23] X. Cheng, X. Huang, D. Li, W. Wu, and D. Du, "A polynomial-time approximation scheme for the minimum-connected dominating set in ad hoc wireless networks," Networks, vol. 42, no. 4, pp. 202-208, 2003.

[24] C. Ambuhl, T. Erlebach, M. Mihalak, and M. Nunkesser, "Constantfactor approximation for minimum-weight (connected) dominating sets in unit disk graphs," in Proc. of the 9th International Workshop on Approximation Algorithms for Combinatorial Optimization Problems, (Barcelona, Spain), August 2006.

[25] D. Hochbaum and W. Maass, "Approximation schemes for covering and packing problems in image processing and VLSI," Journal of the ACM, no. 1, pp. 130-136, 1985.

[26] H. Brönnimann and M. T. Goodrich, "Almost optimal set covers in finite vc-dimension.," Discrete \& Computational Geometry, vol. 14, no. 4, pp. 463-479, 1995.

[27] F. Ingelrest and D. Simplot-Ryl, "Localized broadcast incremental power protocol for wireless ad hoc networks," in Proceedings of 10th IEEE Symposium on Computers and Communications, (La Manga del Mar Menor, Spain), June 2005

[28] X.-Y. Li, "Algorithmic, geometric and graphs issues in wireless networks," Wireless Communications nad Mobile Computing, vol. 3, pp. 119-140, March 2003.

[29] L. Feeney, "An energy-cosumption model for performance analysis of routing protocols for mobile ad hoc networks," ACM Journal of Mobile Netowrks and Applications, vol. 3, no. 6, pp. 239-249, 2001.

[30] G. Calinescu, "Computing 2-hop neighborhoods in ad hoc wireless networks," in 2nd Int. Conference on Ad-Hoc, Mobile, and Wireless Networks, (Montreal, Canada), October 2003.

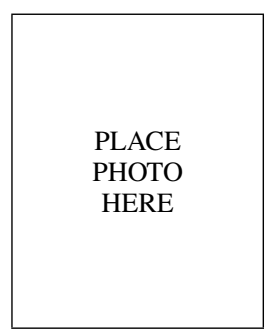

Mehmet Baysan received M.S. and Ph.D. degrees in computer science from The University of Texas at Dallas in 2005 and 2008. He is currently a postdoctoral researcher in Department of Management at University of Toronto. His research interests include algorithms, combinatorial optimization, graph theory and wireless network problems related with these fields.

PLACE

PHOTO

HERE

PLACE

PHOTO

HERE

PLACE

PHOTO

HERE
Kamil Sarac is an Assistant Professor of computer science at the University of Texas at Dallas. He obtained his Ph.D. degree in computer science from the University of California Santa Barbara in 2002. His research interests include network and service monitoring and Internet measurements; energy efficiency in ad hoc networks; overlay networks and their use in network security and denial-of-service defense; and multicast communication.

R. Chandrasekaran is currently Ashbel Smith Professor of Computer Science and Operations Research at The University of Texas at Dallas. He received his $\mathrm{PhD}$ from the University of California at Berkeley. He has served as a faculty member at Case Western Reserve University and been a visitor at Northwestern and Tel Aviv Universities. His research interests are in combinatorial optimization, computational geometry, and integer programming with applications to telecommunication networks.

Sergey Bereg received Ph.D. degree from Minsk Institute of Mathematics, Belarus in 1992. Dr. Bereg joined the Department of Computer Science at the University of Texas at Dallas in 2002 as an Associate Professor. His area of research is in Computational Geometry, Networks and Communications, Computational Biology. 